\title{
A two-level atom in the field of a gravitational wave - on the possibility of parametric resonance
}

\author{
S. V. Siparov \\ Dept. of Physics, Academy of Civil Aviation, 38 Pilotov str., St-Petersburg 196210, Russia \\ Received 21 February 2003 / Accepted 20 October 2003

\begin{abstract}
We consider the influence of a gravitational wave on the dynamics and radiation properties of a two-level atom in the spectroscopically strong resonant electromagnetic field of a cosmic maser. It is shown that the time dependence of the space-time metric can lead to parametric resonance. Such resonance could reveal itself through the appearance of a nonstationary (periodic) component in the observed spectrum of the maser. The amplitude of the nonstationary component is essentially higher than that of the stationary radiation peak corresponding to the additional wave originating from the action of the gravitational wave on the atom associated with the maser. The cosmic maser located in the vicinity of a periodic source of gravitational waves must satisfy the specific demands formulated in the paper. Provided the parametric resonance conditions are fulfilled, the effect of the opto-metrical parametric resonance can be used for the direct registration of gravitational waves with the help of a remote quantum detector - cosmic maser.
\end{abstract}

Key words. gravitational waves

\section{Introduction}

The interferometric methods of gravitational wave $(\mathrm{GW})$ detection are nowadays considered to be the most promising. The idea on which they are based is the following. A monochromatic electromagnetic wave (EMW) is emitted by a laser, it is reflected by a material body (e.g., a mirror) and then falls on the detector. If the emission and detection points coincide (Fig. 1a), then the round travel time of the signal multiplied by the light speed will give twice the distance to the body. If the GW falls upon the system, the distance between the light-source detector and the mirror changes periodically. If this distance is much less than the length of the GW acting on them, and if the GW wave vector is orthogonal to the light beam, then the measured distance can be obtained with the help of the geodesic declination equation (Amaldi \& Pizzella 1979)

$\frac{\mathrm{d}^{2} x^{j}}{\mathrm{~d} t^{2}}-\frac{1}{2 c^{2}} \frac{\partial^{2} h_{i}^{j \mathrm{TT}}}{\partial t^{2}} x^{i}=0$

where $h_{i}^{j \mathrm{TT}}$ is the amplitude of the GW in the traceless transversal frame. The change in distance between the mirror and the detector will be accompanied by a change in the detected signal phase. The registration of the phase change, which is proportional to the GW amplitude will thus give evidence of the existence of GW. This change is very small and is comparable to the noise factors.

This is not the only way in which GW can be detected. The problem of the influence of GW on the structure of the levels of the quantum objects was discussed in detail in Leen et al. (1983). Particularly, they regarded the GW action on the low levels and on the high (Rydberg) levels of a hydrogen-type atom, on the classic oscillator levels and on the rigid rotator levels. The obtained shifts appeared to be extremely small and to have a quadrupole origin, which suggested that the dipole transitions characterizing the system would dominate. Nevertheless, the possibility of using this effect in the remote quantum detectors of the GW radiation was analyzed.

Another method of GW detection was discussed in Pegoraro et al. (1978), where they considered the GW action on an EMW. The proposed device presented two orthogonal electromagnetic resonators tuned in such a way that their frequencies differed by a small value. This value had to coincide with the EMW electric stress change due to the GW field. It was suggested that the periodical change of the populations of the resonators would indicate $\mathrm{GW}$ action.

Send offprint requests to: S. V. Siparov,

e-mail: sergey@siparov.spb.su 


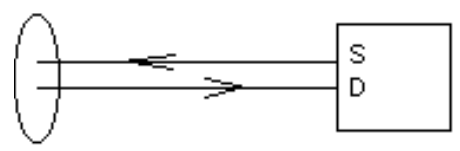

la

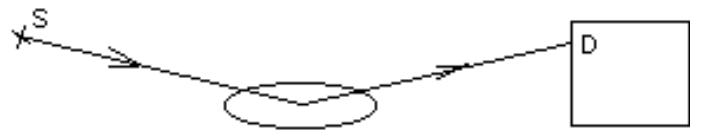

$1 \mathrm{~b}$

Fig. 1. a) Traditional scheme for the interferometric detection of the GW. b) Modified scheme for the interferometric detection of the GW.

Let us first discuss a possible modification of the interferometric method mentioned above. Suppose that the monochromatic EMW source is located on the opposite side of the mirror with reference to the detector, while the reflected signal reaches the detector as before (Fig. 1b). If there is a GW field, the phase of the signal will change proportionally to the GW amplitude in the same way as in the case in Fig. 1a, and such an experimental set up could be also used to detect the GW.

Let us now increase the distance between the "EMW-source-mirror" block and the detector. If the mirror is located sufficiently close to the GW source (e.g., a neutron star), the periodical phase change will become much larger than in the local experiments of the types in Figs. 1a,b. In this case the distance between the mirror and the detector becomes very large and it is impossible to use the geodesic declination formula any longer. However, the period of this phase change will still be equal to the GW period, and, consequently, the phase of the detected signal will still change with the GW period too.

The experiment in Fig. $1 \mathrm{~b}$ is an analogue to the situation where a body vibrates relative to a detector for an arbitrary reason and an EMW is scattered by this body. In this case the detected signal shows a periodic phase shift. The analogy will be complete if the amplitude of the vibrations is equal to the GW amplitude at the location of the body and the period of the vibrations is equal to the period of the GW.

The interpretation of the interferometric experiments, (Fig. 1a), for obvious reasons does not include the frequency variation of the EMW due to GW action (Amaldi \& Pizzella 1979). However, if the experiment is performed with the help of a remote detector as mentioned above, one could analyze other possible changes caused by the GW action on the EMW, similar to those discussed in Pegoraro et al. (1978). In the experiments in Fig. 1b, instead of a mirror one could use any object capable of interacting with the monochromatic EMW. For example, there could be an atom which has a transition resonant to the EMW. The necessary electromagnetic radiation can be supplied by the cosmic maser associated with this atom.

At this point, it is useful to include in the discussion the situations investigated by Siparov (1997 - Paper I, and 1998 Paper II). The system considered there presents an atom which has only two levels (this model is frequently and effectively used in theoretical spectroscopy), which is placed into a resonant laser field and which is mechanically vibrating for some additional reason. Provided certain conditions are fulfilled, this system can reveal the parametric resonances (PR) due to the fact that it contains several frequency-dependent parameters. One of them is the Rabi frequency of the external EMW field acting on the atom; this Rabi frequency is proportional to the intensity of the EMW. The other, (see Papers I and II), is the frequency of the mechanical vibrations of an atom. In the problem under discussion, this other parameter is the GW frequency. In Papers I and II it was shown that the PR can lead to the appearance of nonstationary components in the observable spectra of scattered radiation. It was also shown that the amplitudes of these nonstationary components are larger than the regular (stationary) components of the spectra. To observe these features in the experiment, the set-up should include a frequency filter tuned at the frequency of the atomic vibrations. In Papers I and II where these features were caused by the mechanical oscillations of the atom, the effect was called opto-mechanical parametric resonance.

To apply these ideas to the atom of a cosmic maser in the GW field, it is necessary to analyze the system consisting of the atom producing the maser, the field of the maser, the GW source and the detector on the Earth and to make sure that the PR is possible. In Siparov (2001 - Paper III) it was shown that for a carefully selected cosmic maser the PR conditions formulated in Papers I and II can be fulfilled. This means that in this case the regular signal from the cosmic maser which is well observed on the Earth (Cook 1977; Elitzur 1992) could acquire a GW-caused nonstationary component which would also be observable. Since this effect would be caused by the change in the metric, it may be called opto-metrical PR.

The discussion goes as follows. Let us consider a particle which is a two-level atom interacting with the spectroscopically strong EMW resonant to the transition ("spectroscopically strong" means that the radiation from the atom is dominated by the stimulated radiation, viz. the underlying radiation could be weak). The GW action on this system can reveal itself through a) the transformation of the states of the atom, b) the modification of the EMW, and c) the periodical displacement of the particle as a whole. First, the GW action on the radiative properties of the atom will be discussed. Then the change in the EMW characteristics will be taken into account. Then the particle motion in the GW field will be described. The results obtained will make it possible to formulate and solve the PR problem. The last section discusses the physical implications of the results.

\section{GW action on the structure of the atomic levels}

The Schrödinger equation for our case is

$i \hbar \frac{\mathrm{d}}{\mathrm{d} t} \Psi=H \Psi$ 
The Hamiltonian $H$ here is

$H(t)=H_{0}+H_{\mathrm{g}}(t)+H_{\mathrm{em}}(t)$

where $H_{0}$ is the atomic Hamiltonian which is independent of time, $H_{\mathrm{g}}(t)$ is the perturbation of the atomic Hamiltonian due to $\mathrm{GW}$-atom interaction, $H_{\mathrm{em}}(t)$ describes the EMW-atom dipole interaction which can be written as usual

$H_{\mathrm{em}}(t)=\mu E$.

Here $\mu$ is a matrix element of the induced dipole moment operator, $E=E(t)$ is the electric stress of the EMW. The distance between the EMW resonant levels is much larger than the distance between the sublevels that could appear because of the GW (Leen et al. 1983). Then $\mu$ can be calculated with the help of the perturbation theory using the corrections to the wave functions which appear due to the GW action. Therefore, let us first find the wave functions of the perturbed Hamiltonian

$i \hbar \frac{\mathrm{d}}{\mathrm{d} t} \Psi^{(1)}=H_{1} \Psi=\left[H_{0}+H_{\mathrm{g}}(t)\right] \Psi^{(1)}$.

The relativistic calculation performed in Leen et al. (1983) gives ${ }^{1}$

$H_{\mathrm{g}}=m D\left(\frac{G L}{4 c^{3}}\right)^{1 / 2}\left[\epsilon_{i j}^{+}+\epsilon_{i j}^{\times}\right] X^{i} X^{j} \frac{\cos \left(K_{\mathrm{g}} r_{\mathrm{S}}-D t\right)}{r_{\mathrm{S}}}$.

Equation (6) may be rewritten as follows

$H_{\mathrm{g}}=F \mathrm{e}^{-i D t}+G \mathrm{e}^{i D t}$

$F=\frac{m D}{2 r_{\mathrm{S}}}\left(\frac{G L}{4 c^{3}}\right)^{1 / 2}\left[\epsilon_{i j}^{+}+\epsilon_{i j}^{\times}\right] X^{i} X^{j} \mathrm{e}^{i K_{\mathrm{g}} r_{\mathrm{S}}}$

$F_{n m}=\int\left(\Psi_{n}^{(0)}\right)^{*} F\left(\Psi_{m}^{(0)}\right) \mathrm{d} q ; F_{n m}=G_{m n}^{*}$

where $F$ and $G$ do not depend on time. Since $F$ is obviously small (see the footnote), let us use the perturbation theory for the case where the perturbations are periodic functions of time (Landau \& Lifshits 1963). The first approximation for the wave functions gives

$\Psi_{n}^{(1)}=\Psi_{n}^{(0)}+\sum_{k} a_{k n}(t) \Psi_{k}^{(0)}$

where $\Psi_{m}^{(0)}$ are the wave functions of the unperturbed Hamiltonian, and

$a_{k n}=-\frac{F_{k n} \mathrm{e}^{i\left(\omega_{k n}-D\right) t}}{\hbar\left(\omega_{k n}-D\right)}-\frac{F_{n k}^{+} \mathrm{e}^{i\left(\omega_{k n}+D\right) t}}{\hbar\left(\omega_{k n}+D\right)}$.

Equations (8) and (9) describe the wave functions of an atom in the GW field.

The expression for the induced dipole moment is

$\mu=\left(1+a_{11}+a_{22}\right) \mu_{0}+e \int \sum_{k \geq 2} a_{k 1}\left(\Psi_{n}^{(0)}\right)^{*} r\left(\Psi_{2}^{(0)}\right) \mathrm{d} q+e \int \sum_{k \neq 2} a_{k 2}\left(\Psi_{1}^{(0)}\right)^{*} r\left(\Psi_{k}^{(0)}\right) \mathrm{d} q$

where $\mu_{0}$ is the dipole moment calculated with the wave functions $\Psi_{1}^{(0)}, \Psi_{2}^{(0)}$ of the unperturbed Hamiltonian. As follows from Eq. (9), the expressions for $a_{11}$ and $a_{22}$ are

$a_{11}=a_{22}=\frac{1}{\hbar D}\left(F_{11} \mathrm{e}^{-i D t}-F_{22}^{+} \mathrm{e}^{i D t}\right)$.

Now let only one pair of levels take part in the electromagnetic interaction, that is let us use the two-level model of an atom. Let the frequency of the external monochromatic field correspond to the 1-2 transition. Then Eq. (9) means that the two last terms in Eq. (10) are proportional to the factors oscillating at high frequency; therefore they may be omitted in the further calculation of the dipole moment.

${ }^{1}$ Here $D$ is the GW frequency, $m$ is the electron mass, $G$ is the gravitation constant, $L$ is the full energy flow from a GW source, $c$ is the light speed, $X^{i}$ is the coordinate components in the atomic center of inertia frame, $K_{\mathrm{g}}$ is the GW wave vector, $r_{\mathrm{S}}$ is the distance from the atom to the GW source, $\epsilon_{i j}^{+}=\left(\begin{array}{cc}1 & 0 \\ 0 & -1\end{array}\right), \epsilon_{i j}^{\times}=\left(\begin{array}{ll}0 & 1 \\ 1 & 0\end{array}\right)$ are the unity polarization tensors with nonvanishing components in the directions orthogonal to the GW propagation. 
The evaluation of the correction to the dipole moment caused by the GW gives

$a_{11}=\frac{1}{\hbar D} F \simeq \frac{1}{\hbar D} \frac{m D}{2 r_{\mathrm{S}}}\left(\frac{G L}{4 c^{3}}\right)^{1 / 2} r_{\mathrm{a}}^{2}$

where $r_{\mathrm{a}}$ is the radius of the atom. To calculate the gravitational energy flow, $L$, falling on the atom, let us use (Amaldi \& Pizzella 1979) the formula

$L=\frac{288 G I^{2} g_{\mathrm{e}}^{2} D^{6}}{45 c^{5}}$

characterizing a neutron star with gravitational ellipticity $g_{\mathrm{e}}$ and moment of inertia $I$. Since the expression (Thorne 1987 ) for the dimensionless GW amplitude $h$ is

$h=\frac{G M R^{2} D^{2} g_{\mathrm{e}}}{c^{4} r_{\mathrm{S}}}$

where $R$ is the radius of the star, Eq. (12) gives

$a_{11}=\frac{1}{\hbar D} 12 \sqrt{\frac{2}{5}} D^{2} m r_{\mathrm{a}}^{2} h=12 \sqrt{\frac{2}{5}} \frac{m r_{\mathrm{a}}^{2}}{\hbar} D h$.

The correction to the induced dipole moment is linear in $h$, the coefficient is defined by Eq. (15) in which $m$ and $r_{\mathrm{a}}$ are the atomic mass and radius.

\section{The GW action on the EMW}

To describe the wave process, it is convenient to use the eikonal, i.e. the phase of the propagating wave $\mathrm{e}^{i \psi}$. The EMW eikonal equation in flat space-time (no gravitational field) can be written as

$\frac{\partial \psi}{\partial x_{i}} \frac{\partial \psi}{\partial x^{j}}=0$

In the simplest case the eikonal is constant

$\psi=\omega t-\boldsymbol{k} \cdot \boldsymbol{r}$

where $\omega$ and $\boldsymbol{k}$ are the frequency and the wave vector of the EMW. 1967)

Rewriting Eq. (16) for the case when there is a gravitation field and the space-time is curved, one gets (Landau \& Lifshits

$g^{i k} \frac{\partial \psi}{\partial x^{i}} \frac{\partial \psi}{\partial x^{k}}=0$

where $g^{i k}$ is the metric tensor. In the case when the GW propagates in the $x$ (i.e. $x^{1}$ ) direction

$g^{i k}=\left(\begin{array}{cccc}1 & 0 & 0 & 0 \\ 0 & -1 & 0 & 0 \\ 0 & 0 & -1+h \cos \frac{D}{c}\left(x^{0}-x^{1}\right) & 0 \\ 0 & 0 & 0 & -1-h \cos \frac{D}{c}\left(x^{0}-x^{1}\right)\end{array}\right)$.

Strictly speaking, this expression corresponds to the GW in vacuum. It will be used here for the same reasons as in the traditional (interferometric) approach, when the laser fields are even larger than those in a cosmic maser and the masses of the parts of the instrument are larger than that of an atom. These reasons take into account the fact that the energy density in the linearized theory of gravitation is of a higher order (see the corresponding discussion in Isaacson 1968; Srevin et al. 2001; Papadopoulos 2002a,b).

In Eq. (19) $h \ll 1$ is again the nondimensional amplitude of the GW, and the GW polarization is considered to be circular (not elliptical) for simplicity. The eikonal equation has the explicit form

$\left(\frac{\partial \psi}{\partial x^{0}}\right)^{2}-\left(\frac{\partial \psi}{\partial x^{1}}\right)^{2}-\left(\frac{\partial \psi}{\partial x^{2}}\right)^{2}-\left(\frac{\partial \psi}{\partial x^{3}}\right)^{2}+h\left[\left(\frac{\partial \psi}{\partial x^{2}}\right)^{2}-\left(\frac{\partial \psi}{\partial x^{3}}\right)^{2}\right] \cos \frac{D}{c}\left(x^{0}-x^{1}\right)=0$

Since $h \ll 1$, the eikonal can be expressed as

$\psi=f+h g$ 
where $f$ and $g$ are new functions. If we substitute Eq. (21) into Eq. (20), neglect all the terms containing $h$ in the powers higher than unity and consider separately the equations with and without $h$, then

$\left(\frac{\partial f}{\partial x^{0}}\right)^{2}-\sum_{i}\left(\frac{\partial f}{\partial x^{i}}\right)^{2}=0$

$2 \frac{\partial f}{\partial x^{0}} \frac{\partial g}{\partial x^{0}}-2 \frac{\partial f}{\partial x^{i}} \frac{\partial g}{\partial x^{i}}+\left[\left(\frac{\partial f}{\partial x^{2}}\right)^{2}-\left(\frac{\partial f}{\partial x^{3}}\right)^{2}\right] \cos \frac{D}{c}\left(x^{0}-x^{1}\right)=0$.

The first of Eqs. (22) gives

$\omega^{2}=c^{2} k^{2}$

which means that the principal term of the EMW eikonal in the GW field corresponds to a flat wave. Substituting this result into the second equation of Eqs. (22), one gets the equation for the function $g$

$2 \frac{\omega}{c} \frac{\partial g}{\partial x^{0}}+2 k_{1} \frac{\partial g}{\partial x^{1}}+2 k_{2} \frac{\partial g}{\partial x^{2}}+2 k_{3} \frac{\partial g}{\partial x^{3}}+\left(k_{2}^{2}-k_{3}^{2}\right) \cos \frac{D}{c}\left(x^{0}-x^{1}\right)=0$.

This is a first-order quasilinear inhomogeneous partial differential equation. Its solution is

$g=-c^{2} \frac{k_{2}^{2}-k_{3}^{2}}{2 \omega D} \cos \frac{D}{c}\left(x^{0}-x^{1}\right)+\varphi\left(k_{1} x^{0}-\frac{\omega}{c} x^{1}, k_{2} x^{1}-k_{1} x^{2}, k_{3} x^{2}-k_{2} x^{3}\right)$

where $\varphi$ is an arbitrary function defined by the boundary conditions. This function can be taken equal to zero for simplicity.

Finally, the eikonal for the case when the GW acts on the EMW can be written as

$\psi=\frac{\omega}{c} x^{0}-k_{i} x^{i}-h c^{2} \frac{k_{2}^{2}-k_{3}^{2}}{2 \omega D} \cos \frac{D}{c}\left(x^{0}-x^{1}\right)$.

These calculations show that the GW action upon the EMW is equivalent to an EMW phase modulation with the frequency of the GW and amplitude

$A_{\mathrm{em}}=h \frac{\omega}{D}$

\section{A free particle in the GW field}

Let us now describe the behavior of a particle in the GW field with the help of the geodesic equation. Our aim is to evaluate the amplitude of the particle's periodic displacement orthogonal to the GW wave vector.

As an introductory example of the influence of the displacement of the particle on the EMW signal emitted by the particle, let us consider the following situation. Let a motionless particle located in the coordinate origin transmit periodic electromagnetic signals to the remote detector. Then the intervals of time between the consecutively emitted signals, $\Delta t_{\mathrm{p}}$, are equal to the intervals of time, $\Delta t_{\mathrm{d}}$, between the consecutively signals received by the detector. But if the particle vibrates in parallel to the emitted EMW according to a sine law, ( $D$ is the frequency of these vibrations, $\eta$ is the amplitude), then the intervals of time between the consecutively received signals are expressed by the intervals of time between the consecutively emitted signals by

$\Delta t_{\mathrm{p}}=\Delta t_{\mathrm{d}}+\frac{k \eta}{\omega} \sin \left(D \Delta t_{\mathrm{p}}\right)$

This means that the eikonal suffers a periodic time shift.

Let the location of the atom be in the coordinate origin, the $x^{1}$ axis is the GW wave vector direction, the $x^{2}$ axis is the EMW wave vector direction coinciding with the direction at the Earth. The distance between the atom and the GW source is much smaller than the distance from any of them to the Earth. The maser field will be considered to be absent. Then the particle in the gravitation field moves along the geodesic described by the equation (Landau \& Lifshits 1967)

$\frac{\mathrm{d}^{2} x^{i}}{\mathrm{~d} s^{2}}+\Gamma_{k l}^{i} \frac{\mathrm{d} x^{k}}{\mathrm{~d} s} \frac{\mathrm{d} x^{l}}{\mathrm{~d} s}=0$

The Christoffel coefficients $\Gamma_{k l}^{i}$ can be expressed with the help of the metric tensor components

$\Gamma_{k l}^{i}=\frac{1}{2} g^{i m}\left(\frac{\partial g_{m k}}{\partial x^{l}}+\frac{\partial g_{m l}}{\partial x^{k}}-\frac{\partial g_{k l}}{\partial x^{m}}\right)$. 
Calculating the coefficients $\Gamma_{k l}^{i}$ with regard to Eq. (19) for the GW metric tensor, one can substitute them all into the Eq. (29), neglect the terms in which the power of $h$ is higher than unity, and obtain the following system of equations

$t^{\prime \prime}+\frac{1}{2} h \frac{D}{c^{2}}\left(y^{\prime 2}-z^{\prime 2}\right) \sin \frac{D}{c}(c t-x)=0$.

$x^{\prime \prime}+\frac{1}{2} h \frac{D}{c}\left(y^{\prime 2}-z^{\prime 2}\right) \sin \frac{D}{c}(c t-x)=0$

$y^{\prime \prime}+\frac{1}{2} h \frac{D}{c} y^{\prime}\left(c t^{\prime}-x^{\prime}\right) \sin \frac{D}{c}(c t-x)=0$

$z^{\prime \prime}+\frac{1}{2} h \frac{D}{c} z^{\prime}\left(c t^{\prime}-x^{\prime}\right) \sin \frac{D}{c}(c t-x)=0$.

Here the common notation $t=x^{0} / c, x=x^{1}, y=x^{2}, z=x^{3}$ was used again and $w^{\prime} \equiv \frac{\mathrm{d} w}{\mathrm{~d} s}$. The first two equations of the system Eq. (31) give

$c t^{\prime \prime}=x^{\prime \prime}$

which leads to

$x=c t+a s+b$

where $a$ and $b$ are arbitrary constants.

Let us analyze the meaning of the last expression. At the moment $t=0$ the atom is motionless in the coordinate origin of the frame relative to the GW source center, i.e. $x=0, s=0$. That is why $b=0$. If there were no gravity field, the atom would never leave its place, therefore, $s=-c t / a, a=1$. But, since there is a GW, the value of $a$ in view of the linear approximation used might differ from unity by a value proportional to $h$. That is $a=1+h a_{1}$ where $a_{1}=O(1)$, and Eq. (33) gives

$x=c t+\left(1+h a_{1}\right) s$.

Substitute this expression into the third equation of the system Eq. (31) corresponding to the direction towards the Earth. Neglecting the terms in which the power of $h$ is higher than unity, one gets

$y^{\prime \prime}+h \frac{D}{c} y^{\prime} \sin \left[\frac{D}{c}\left(1+h a_{1}\right) s\right]=0$.

The solution of this equation with regard to $h \ll 1$ is

$y=u_{0} s+h \frac{1}{\left(1+h a_{1}\right)^{2}} \frac{c}{D} \sin \left[\frac{D}{c}\left(1+h a_{1}\right) s\right]+d$

where $u_{0}$ and $d$ are the integration constants. Since $s=\frac{x-c t}{1+h a_{1}}$ and the particle is at the coordinate origin, at $t=0$,

$y=\frac{u_{0}(x-c t)}{1+h a_{1}}-h \frac{1}{\left(1+h a_{1}\right)^{2}} \frac{c}{D} \sin \frac{D}{c}(c t-x)$.

There is a sine dependence of the space coordinate of the atom parallel to the EMW wave vector on the time coordinate of the atom. The amplitude $\eta$ of the corresponding oscillations of the particle in the GW field is

$\eta=h \frac{1}{\left(1+h a_{1}\right)^{2}} \frac{c}{D} \simeq h \frac{c}{D}$

Let us compare these results with Eq. (28). The particle that transmits the signal is influenced by the GW in such a way that there is a periodical phase shift in the signals received by the detector. The frequency of the shift is equal to the GW frequency, and its amplitude is equal to

$k \eta=\frac{\omega}{c} h \frac{c}{D}=h \frac{\omega}{D}$.

Note that the comparison of Eqs. (37) and (39) with Eqs. (28), (26) and (27) shows that both frequencies and amplitudes of the GW action on the EMW and on the behavior of the atom are the same. 


\section{The possibility of parametric resonance}

To be able to investigate the parametric resonance possibility, let us formulate the obtained results and assumptions used.

Equation (15) shows that the GW action on the reconstruction of the atomic levels leading to the change in the dipole interaction is negligible in comparison to the other two effects, defined by Eqs. (27) and (39). Therefore, no additional terms are needed in the expression for the dipole moment in Bloch's equations for the density matrix components corresponding to a two-level atom in a strong electromagnetic field in the $\mathrm{GW}$.

The weak gravitational perturbation of the monochromatic EMW leads to the latter's phase modulation and can be described in terms of the amplitude modulation

$E(t)=E_{0}\left(1-h \frac{\omega}{D} \cos D t\right) \cos \Omega t$.

In these terms it means that the EMW acquires the second component caused by the GW. The first (main) component corresponds to the regular maser radiation which is spectroscopically strong. It can be described by

$E_{1}(t)=E_{0} \cos \left(\Omega_{1} t-k y\right)$

where $\Omega_{1}=\Omega \cong \omega$ is the frequency of the maser field coinciding with the atomic transition frequency. The second (additional) component is much weaker than the first. It can be described by

$E_{2}(t)=E_{0} h \frac{\omega}{D} \cos \left(\Omega_{2} t-k y\right)$

where $\Omega_{2}=\Omega-D$.

The GW wave vector is considered to be orthogonal to the EMW wave vector. In view of the results of the previous section, the GW action on the atomic motion can be interpreted as the appearance of a periodic component $V_{\text {osc }}$ in the component of the atomic velocity parallel to the EMW wave vector. According to Eq. (37)

$V_{\text {osc }}=-V_{1} \cos D t$

$V_{1}=h c$.

In this case the total time derivative can be written as

$\frac{\mathrm{d}}{\mathrm{d} t}=\frac{\partial}{\partial t}+V \frac{\partial}{\partial y}=v-V_{1} \cos D t$.

The nature of the electromagnetic field of a cosmic maser is discussed in Cook (1977) and Elitzur (1992). Here it is important that this field can be described classically. The dynamics of the atom can be described by the density matrix $\rho(t, y)$, and the situation becomes the same as in Papers I and II. The lower level of the atom will be considered the ground level for simplicity. Then Bloch's equations are

$\frac{\mathrm{d}}{\mathrm{d} t} \rho_{22}=-\gamma \rho_{22}+2 i\left[\alpha_{1} \cos \left(\Omega_{1} t-k_{1} y\right)+\alpha_{2} \cos \left(\Omega_{2} t-k_{2} y\right)\right]\left(\rho_{21}-\rho_{12}\right)$

$\frac{\mathrm{d}}{\mathrm{d} t} \rho_{12}=-\left(\gamma_{12}+i \omega\right) \rho_{12}-2 i\left[\alpha_{1} \cos \left(\Omega_{1} t-k_{1} y\right)+\alpha_{2} \cos \left(\Omega_{2} t-k_{2} y\right)\right]\left(\rho_{22}-\rho_{11}\right)$

$\rho_{22}+\rho_{11}=1$.

Here $\rho_{22}$ and $\rho_{11}$ are the populations of the levels, $\gamma$ and $\gamma_{12}$ are the longitudinal and transversal decay rates of the atom (since level 1 is the ground level, $\left.\gamma_{12}=\gamma / 2\right) ; \alpha_{1}=\frac{\mu E_{0}}{\hbar}, \alpha_{2}=\frac{\mu E_{0} h \omega}{D \hbar}$ are Rabi parameters of the main and additional components of the electromagnetic field; $k_{1}$ and $k_{2}$ are the wave vectors of these components. In our case they can be considered equal and equal to $k$. The usual way to deal with a problem like the one expressed by Eq. (45) is to use the rotating wave approximation (RWA). The RWA substitutions give

$\rho_{21}=R_{21} \mathrm{e}^{i\left(\Omega_{1} t-k y\right)}$

$\rho_{12}=R_{12} \mathrm{e}^{-i\left(\Omega_{1} t-k y\right)}$

$\rho_{22}=2^{-1 / 2} \widetilde{\rho_{22}}$.

The main goal now is to obtain the expression for $\operatorname{Im}\left(R_{21}\right)$ which is proportional to the absorption coefficient of the additional EMW caused by the GW. Rewriting Eqs. (45), one gets

$\frac{\mathrm{d}}{\mathrm{d} t} 2^{-1 / 2} \widetilde{\rho_{22}}=-2^{-1 / 2} \gamma \widetilde{\rho_{22}}-i R_{12} \alpha_{1}+i R_{21} \alpha_{1}-i R_{12} \alpha_{2} \mathrm{e}^{i\left(-\left(\Omega_{1}-\Omega_{2}\right) t-k y\right)}+i R_{21} \alpha_{2} \mathrm{e}^{\left.i\left(\Omega_{1}-\Omega_{2}\right) t+k y\right)}$

$\frac{\partial}{\partial t} R_{12}=-\left\{\gamma_{12}+i \omega-i \Omega_{1}+i k\left[V_{0}-V_{1} \cos \left(\left(\Omega_{1}-\Omega_{2}\right) t+k y\right)\right]\right\} R_{12}-i\left[\alpha_{1}+\alpha_{2} \mathrm{e}^{i\left(\left(\Omega_{1}-\Omega_{2}\right) t+k y\right)}\right]\left(2^{1 / 2} \widetilde{\rho_{22}}-1\right)$ 
where $V_{1}=h c$. Now dividing Eqs. (47) by $\alpha_{1}$ and introducing the notation given in the Appendix, one can rewrite Eq. (47) in matrix form

$\frac{\partial}{\partial \tau} W=\left(Q_{0}+\varepsilon Q_{1}(\tau)\right) W+C(\tau)$

where

$Q_{0}=i\left(\begin{array}{ccc}0 & -1 & 1 \\ -1 & -\sigma & 0 \\ 1 & 0 & \sigma\end{array}\right) ; W=\left(\begin{array}{l}\widetilde{\rho_{22}} \\ R_{12} \\ R_{21}\end{array}\right) ; C=i \sqrt{2}\left(\begin{array}{c}0 \\ 1+\varepsilon a \mathrm{e}^{-i \delta_{d} \tau} \\ -1-\varepsilon a \mathrm{e}^{i \delta_{d} \tau}\end{array}\right)$

$Q_{1}=\left(\begin{array}{ccc}-1 & -i a \mathrm{e}^{i \delta_{d} \tau} & i a \mathrm{e}^{-i \delta_{d} \tau} \\ -i a \mathrm{e}^{-i \delta_{d} \tau} & -\Gamma+i \kappa v_{1} \cos \left(\delta_{d} \tau\right) & 0 \\ i a \mathrm{e}^{i \delta_{d} \tau} & 0 & -\Gamma-i \kappa v_{1} \cos \left(\delta_{d} \tau\right)\end{array}\right) ; \sigma=\delta+\kappa v$.

Equation (47) can be solved by the asymptotic expansion method with $\varepsilon$ as a small parameter. Here $\varepsilon$ characterizes the spectroscopic strength of the maser field. The goal is to obtain the principal asymptotic term which defines the absorption coefficient behavior. The mathematical methods used for similar problems are discussed in detail in Kazakov (1992), Kazakov \& Siparov (1997), and Papers I and II. Here it should be stressed that the main point of the solution is the use of the PR conditions

$$
\begin{aligned}
& \sqrt{\sigma^{2}+2}=\left|\delta_{d}\right|+\varepsilon v, v=O(1) \\
& \sqrt{\sigma^{2}+2}=|\delta|+\varepsilon v, v=O(1)
\end{aligned}
$$

that must be fulfilled in order to get the result. Finally, the solution gives

$\operatorname{Im}\left(R_{21}\right) \sim a L[-C+A \cos (D t+\varphi)]$

where the explicit expressions for $L, C, A$ and $\varphi$ are given in the Appendix.

The physical meaning of the obtained result is the following.

First, if there is no gravitation radiation, then $a=0$ (see the notation in the Appendix), and no observable effects due to the $\mathrm{PR}$ appear in the principal term of the asymptotic expansion. Consequently, no additional signal appears in the observable maser signal. Second, if there is a GW and the PR conditions are fulfilled, then the weak additional wave due to the GW action on the EMW is amplified, since the stationary component of the absorption coefficient in Eq. (49) has a negative sign. Third, the principal term of the asymptotic expansion used to calculate the absorption coefficient of the additional wave contains a nonstationary term proportional to A. Similar to the situations discussed in Paper I, Paper II and also in Kazakov (1992) and Kazakov \& Siparov (1997), this means that at the frequency shifted from the maser frequency by the GW frequency the absorption is periodically replaced by amplification. The frequency of the absorption-to-amplification change is equal to the GW frequency. This third result is the most important one and represents the main result of this paper. Whatever structure the stationary and well observed maser signal has, the appearance of a nonstationary component observed with the help of an additional frequency filter will indicate the existence of a parametric resonance in the system. This might then be due to a periodical change in the EMW and in the velocity of the atom.

If the cosmic maser is located in the vicinity of the GW source so that the PR conditions discussed in detail in Paper III are fulfilled, then there are only two possible mechanisms to make the atoms vibrate. One of them is the interaction of the magnetic moment of masering atoms with the alternating magnetic field of the $\mathrm{GW}$ source. If the masering atoms have no magnetic moment, then the only reason for their mechanical vibrations is gravitational radiation.

The conclusion is that if the nonstationary signal discussed above is observed and if it has the GW source frequency, then this gives direct evidence of the existence of $\mathrm{GW}$.

\section{Discussion}

The results obtained in this paper are the following. When the GW acts upon a two-level atom interacting with the spectroscopically strong resonant EMW, three types of corrections can appear in the behavior of the atom. The first correction is due to the change in the dipole moment matrix element described by Eq. (15). The second correction is due to the change of the EMW described by Eqs. (26-27). The third correction is connected with the time dependence of the coordinate of the atom described by Eqs. (37-39). As can be seen from the mentioned formulas, the weak influence of the GW on the reconstruction of the atom levels is negligible in comparison with the two other effects, both of which lead to the appearance of additional terms in the equations used to investigate the situation. It has been shown that the solution of Bloch's equations for the density matrix components of the two-level atom interacting with spectroscopically strong resonant EMW and the GW should make use of the regular dipole moment. The GW action on the monochromatic EMW causes a periodical phase modulation and is comparable to the GW action on the time dependence of coordinate of the atom that is orthogonal to the GW wave vector. If a cosmic maser is chosen such 
that its parameters provide the possibility of the realization of PR, and if the geometry of the maser-GW source-Earth system can also provide the realization of PR (see below), then both these effects could lead to opto-metrical parametric resonance (OMPR), the result of which could be observed with the help of an experimental set up supplied with a frequency filter.

Direct GW detection has been a challenging problem for years (Schutz 1999). Up to now the main evidence of the existence of GW radiation is given in Hulse \& Taylor (1975), and it is indirect evidence. The possibilities provided by the method based on the effect of the OMPR discussed here could lead to the direct registration of the GW signal. The experimental problems characteristic for the suggested method are essentially different from the known problems for the existing methods which are: superhigh sensitivity and superhigh selectivity of the experimental set up. In the case of an OMPR-based method the main difficulty is to find the appropriate astrophysical system, the demands for which are very specific. On the one hand, the upper level of the maser atom transition has to be a metastable one to provide the spectroscopically strong field whose Rabi frequency must coincide with the GW frequency to achieve the PR. On the other hand, the gas clouds in the close vicinities of the neutron stars (which are the periodic GW sources) usually undergo powerful radiation that might prevent the existence of a stable maser radiation region.

In Paper III it was shown that the principal condition defining the location of a cosmic maser able to take part in the OMPR is $h \frac{\omega}{D} \sim \frac{\gamma}{\alpha}$.

For example, for the known OH-masers (Litvak 1972) $\omega \sim 10^{10} \mathrm{~s}^{-1}, \gamma \sim 10^{-1} \mathrm{~s}^{-1}$. If the intensity of the maser is such that the Rabi frequency of its radiation is $\alpha \sim 10^{1} \mathrm{~s}^{-1}$, and the pulsar frequency is $D \sim 10^{2} \mathrm{~s}^{-1}$, then the amplitude of the GW should be $h \sim 10^{-10}$. For the Crab and Vela pulsars this corresponds to a distance $r \sim 10^{5}-10^{6} \mathrm{~m}$ which is close to the border of the wave zone (Paper III).

Nevertheless, the needed system can in principle exist, and this must be considered in the astronomical and astrophysical investigations. The recent discovery of a laser effect in the star shells (Johansson \& Letokhov 2002) essentially broadens the field of search for a suitable astrophysical system.

Acknowledgements. The author expresses his deep gratitude to M.V. Babich for the numerous helpful discussions and to A.Ya. Kazakov and N.A. Razumovskij for the discussions and helpful criticisms.

\section{Appendix}

The notation used in Eqs. (47) and (48).

$2^{1 / 2} \alpha_{1} t=\tau ; \frac{\Omega_{2}-\Omega_{1}}{\alpha_{1}}=2^{1 / 2} \delta_{\mathrm{d}} ; \frac{k}{\alpha_{1}}=2^{1 / 2} \kappa ; \frac{\gamma}{\alpha_{1}}=2^{1 / 2} \varepsilon ; \frac{\gamma_{12}}{\alpha_{1}}=2^{1 / 2} \Gamma \varepsilon ;$

$\frac{\omega-\Omega_{1}}{\alpha_{1}}=2^{1 / 2} \delta ; \frac{\alpha_{2}}{\alpha_{1}}=a \varepsilon ; V_{1}=v_{1} \varepsilon$.

Here $\tau$ is the dimensionless time, $\delta_{\mathrm{d}}$ is the dimensionless shift of the frequencies of the main and additional waves, $\varepsilon$ is the dimensionless small parameter characterizing the spectroscopical strength of the field, $\Gamma$ is the dimensionless transversal decay rate, $\delta$ is the dimensionless shift of the main wave and the atomic transition frequencies, $a$ is the dimensionless amplitude of the additional (weak) wave, $v_{1}$ is the dimensionless velocity due to the atomic vibrations. The $2^{1 / 2}$ factor is not obligatory and is not always used. Here it is introduced to make the form of the equations fit the form used in Kazakov \& Siparov (1997).

The notation used in Eq. (49)

$$
\begin{aligned}
L & =\frac{F+\sigma}{F^{2} \sqrt{2}\left[(\operatorname{Re}(\operatorname{det} K))^{2}+(\operatorname{Im}(\operatorname{det} K))^{2}\right]} \\
C & =\frac{a v(F+\sigma)}{F}\left\{\left(\sigma^{2}+2 \Gamma\right)\left(B^{2}+v^{2}\right)+\frac{2 B F^{2}}{(F-\sigma)^{2}}\left[4 \kappa^{2} v_{1}^{2}(F-\sigma)^{2}+a^{2}\right]\right\} \\
A & =\sqrt{N_{\mathrm{c}}^{2}+N_{\mathrm{s}}^{2}} \\
\varphi & =-\arctan \frac{N_{\mathrm{s}}}{N_{\mathrm{c}}}
\end{aligned}
$$

$\operatorname{Re}(\operatorname{det} K)=\frac{1}{F^{2}}\left\{2 B\left[4 \kappa^{2} v_{1}^{2} F^{2}-\frac{a^{2} F^{2}}{(F-\sigma)^{2}}\right]-\left(\sigma^{2}+2 \Gamma\right)\right\}$

$\operatorname{Im}(\operatorname{det} K)=\frac{1}{F^{2}} \frac{8 a \kappa v_{1} B F^{2}}{F-\sigma}$

$F=\sqrt{\sigma^{2}+2} ; B=1+\Gamma\left(\sigma^{2}+1\right)$ 


$$
\begin{aligned}
N_{\mathrm{c}}= & \frac{1}{F^{2}}\left\{-B F\left(\sigma^{2}+2 \Gamma\right)^{2}\left(B^{2}+v^{2}\right)+2 B^{2} F\left(\sigma^{2}+2 \Gamma\right)\left(4 \kappa^{2} v_{1}^{2} F^{2}-\frac{a^{2} F^{2}}{(F-\sigma)^{2}}\right)\right. \\
& +\sigma\left(8 \kappa^{2} v_{1}^{2} F^{2}-\frac{a^{2} F^{2}}{(F-\sigma)^{2}}\right)\left(\sigma^{2}+2 \Gamma\right)\left(B^{2}+v^{2}\right)-2 \sigma B\left(8 \kappa^{2} v_{1}^{2} F^{2}-\frac{a^{2} F^{2}}{(F-\sigma)^{2}}\right) \\
& \left.\times\left(4 \kappa^{2} v_{1}^{2} F^{2}-\frac{a^{2} F^{2}}{(F-\sigma)^{2}}\right)-\frac{8 a \kappa v_{1} B F^{2}}{F-\sigma}\left[v F\left(\sigma^{2}+2 \Gamma\right)+\frac{8 a \kappa v_{1} B F^{2}}{F-\sigma}\right]\right\} \\
N_{\mathrm{s}}= & -\frac{1}{F^{2}}\left\{[ v \sigma ( \sigma ^ { 2 } + 2 \Gamma ) + \frac { 8 a \kappa v _ { 1 } B F ^ { 3 } } { F - \sigma } ] \left[2 B\left(4 \kappa^{2} v_{1}^{2} F^{2}-\frac{a^{2} F^{2}}{(F-\sigma)^{2}}\right)\right.\right. \\
& \left.-\left(\sigma^{2}+2 \Gamma\right)\left(B^{2}+v^{2}\right)+\frac{8 a \kappa v_{1} B F^{2}}{F-\sigma}\left[B \sigma\left(\sigma^{2}+2 \Gamma\right)+F\left(8 \kappa^{2} v_{1}^{2} F^{2}-\frac{a^{2} F^{2}}{(F-\sigma)^{2}}\right)\right]\right\} .
\end{aligned}
$$

\section{References}

Amaldi, E., \& Pizzella, G. 1979, in Astrofisika e Cosmologia Gravitazione Quanti e Relativita (Firenze, Guinti Barbera) (Russ. Trans. 1982, Mir, Moscow, 241)

Leen, T. K., Parker, L., \& Pimentel, L. O. 1983, Gen. Relat. Gravit., 15, 761

Pegoraro, F., Picasso, E., \& Radicati, L. A. 1978, J. Phys. A, 11, 1949

Siparov, S. V. 1997, Phys. Rev. A, 55, 3704, Paper I

Siparov, S. V. 1998, J. Phys. B, 31, 415, Paper II

Siparov, S. V. 2001, Space-time and Substance, 2, 44, Paper III

Cook, A. H. 1977, Celestial masers (London: Cambridge University Press)

Elitzur, M. 1992, Astronomical masers (Boston: Kluwer Academic Publishers)

Landau, L., \& Lifshits, E. 1963, Quantum mechanics (Moscow: Nauka)

Thorne, K. S. 1987, in Three hundred years of gravitation, ed. S. W. Hawking, \& W. Israel (Cambridge University Press)

Landau, L., \& Lifshits, E. 1967, The theory of field (Moscow: Nauka)

Isaacson, R. A. 1968, Phys. Rev., 166, 1263

Srevin, M., Brodin, G., \& Marklund, M. 2001, Phys. Rev. D, 64, 024013

Papadopoulos, D. B. 2002a, A\&A, 396, 1045

Papadopoulos, D. B. 2002b, Class. Quant. Grav., 19, 2939

Kazakov, A. Ya. 1992, JETP, 102, 1484

Kazakov, A. Ya., \& Siparov, S. V. 1997, Opt. i Spectrosk., 83, 961

Schutz, B. F. 1999, Class. Quant. Grav., 16, A131

Hulse, R. A., \& Taylor, J. H. 1975, ApJ, 195, L51

Litvak, M. M. 1972, in Atoms and Molecules in Astrophysics (London and New York: Academic Press)

Johansson, S., \& Letokhov, V. S. 2002, Pis'ma v ZhETF (rus), 75, 591 